\title{
DYNAMICALLY EQUIVALENT OUTPUTS AND THEIR USE IN NONLINEAR CONTROLLER SYNTHESIS
}

\author{
RAYMOND A. WRIGHT ${ }^{\dagger}$ and COSTAS KRAVARIS \\ Department of Chemical Engineering, University of Michigan, Ann Arbor, MI 48109-2136, USA.
}

(First received 28 January 1992; accepted in revised form 20 September 1992)

\begin{abstract}
A new notion, dynamically equivalent outputs, is developed to aid in controller synthesis for nonlinear systems. A dynamically equivalent output simplifies the controller design problem and, when controlled to its set point, guarantees that the system's primary output is also controlled to its set point. The use of this notion is demonstrated for three classes of systems. For systems with linear state equations but a nonlinear state/output map, conditions are derived for the existence of a linear dynamically equivalent output. Analogous conditions are derived for nonlinear systems for which the state equations can be made linear by means of a coordinate transformation and state feedback. Design of a controller in terms of the new output is straightforward, leading to a response which is nonlinear in the primary output. Simulations for a chemical reactor system using a coordinate transformation, state feedback, and a dynamically equivalent linear output are given. Finally, for general nonlinear systems, the use of input/output linearizing feedback in terms of a dynamically equivalent output is explored. Another chemical reactor system is simulated to demonstrate this approach.
\end{abstract}

\section{INTRODUCTION}

The key idea in a linear inferential control structure is to design a controller, not for the measured output, but for an auxiliary output that must be inferred on-line. This is necessary when it is not practical or physically possible to continuously measure the desired output. In this work, a similar control structure will be used for nonlinear systems. However, the underlying motivation will be to define auxiliary outputs that simplify or in some way aid in the design of the controller.

It is most natural, from a control point of view, to define auxiliary outputs that are "equivalent" in some sense. Loosely speaking, the term equivalent output will be used to refer to an output for a system, other than the primary controlled output of the system, that goes to its set point if and only if the primary output goes to its set point. It is well known from systems theory that by changing the output, both the process zeros (or zero dynamics) and the static gain can be affected. Therefore, it is natural to consider two cases:

(1) Require the equivalent output to have the same static gain as the primary output, but with possibly different zero dynamics (statically equivalent outputs)

(2) Require the equivalent output to have the same zero dynamics as the primary output, but with possibly different static gain (dynamically equivalent outputs).

The gencral control structure which will be developed for use with either type of equivalent output is shown in Fig. 1.

'Present address: Dow Chemical Co., 1400 Building, Midland, MI 48667, U.S.A.
Statically equivalent outputs were defined and their use for nonminimum phase compensation for nonlinear processes was detailed in Wright and Kravaris (1992). The notion of statically equivalent outputs was also useful for deadtime compensation for nonlinear processes (Wright, 1990).

This paper defines the notion of dynamically equivalent outputs. The first, and simplest class of systems for which this type of equivalent output is useful is nonlinear processes in which the only nonlinearity is the static gain of the process, e.g. pH processes. These notions are applied theoretically to $\mathrm{pH}$ processes in Wright and Kravaris (1991), and experimental results are given for a laboratory-scale $\mathrm{pH}$ system in Wright et al. (1991). The second class of systems for which dynamically equivalent outputs are useful are ones where the nonlinear process dynamics meet the Su-Hunt-Meyer (SHM) involutivity conditions. The dynamics of the processes can be made linear via state feedback. However, the process output is typically a nonlinear function of the states in the new coordinate system. Dynamically equivalent outputs will also be applied to a third class of systems where the dynamics are nonlinear, the state/primary output map is nonlinear, and the state/dynamically equivalent output map is also nonlinear. For such systems, where it is assumed that controlling the dynamically equivalent output is physically more meaningful than controlling the primary output, the globally linearizing controller structure (GLC) (Kravaris and Chung, 1987) can be applied. Note that for all three classes of systems, the closed-loop response in terms of the $\mathrm{dy}$ namically equivalent output will be linear, but the closed-loop response of the primary output will be nonlinear.

Throughout this paper, the system under consideration will be a SISO nonlinear system with two 


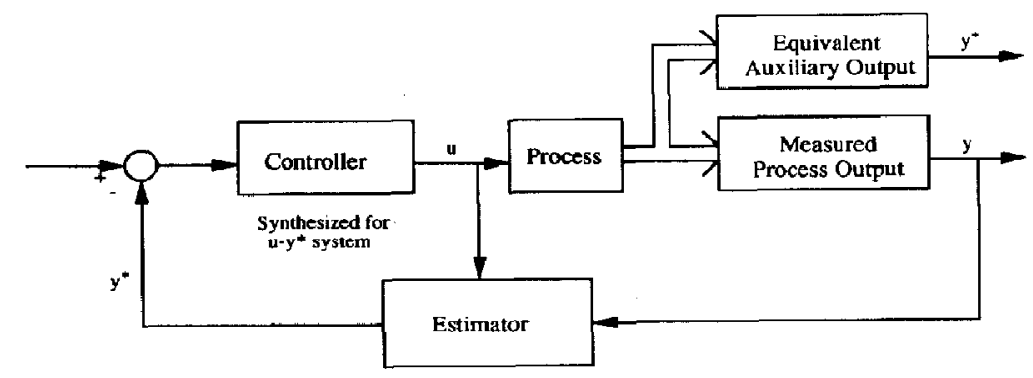

Fig. 1. General output feedback control structure for use with equivalent outputs.

different outputs of the form

$$
\begin{aligned}
\dot{x} & =f(x)+g(x) u \\
y & =h(x) \\
y^{*} & =h^{*}(x)
\end{aligned}
$$

where $u \in \mathbf{R}, x \in \mathbf{R}^{n}, y \in \mathbf{R}$ is the primary process output, and $y^{*} \in \mathbf{R}$ is an auxiliary output. In Section 2 of this paper, the mathematical definition for dynamically equivalent outputs will be given. The use of dynamically equivalent outputs for systems with linear dynamics but a nonlinear state/output map will then be investigated. The combination of dynamically equivalent outputs and SHM linearizing state feedback for systems with nonlinear dynamics will be explored in Section 4. Computer simulations for a nonisothermal CSTR example are used to illustrate this method. Section 5 is concerned with the more general question of synthesizing nonlinear controllers via dynamically equivalent outputs to give nonlinear closed-loop dynamics. A nonisothermal CSTR computer simulation example is also used to demonstrate this result.

\section{DYNAMICALLY EQUIVALENT OUPUTS}

The notion of dynamic output equivalence refers to the case where the auxiliary output has a different static gain but the same zero dynamics. To explore this concept, it is first necessary to define the level sets of the output functions. Given an arbitrary value of the output $y_{0}$, the corresponding level set of $h(x)$ is given by

$$
l_{y n}=\left\{x \in \mathbf{R}^{n} \mid h(x)=y_{0}\right\}
$$

and similarly, given an arbitrary value $y_{0}^{*}$, the corresponding level set of $h^{*}(x)$ is given by

$$
l_{y_{0}^{*}}=\left\{x \in \mathbf{R}^{n} \mid h^{*}(x)=y_{0}^{*}\right\}
$$

There are two ways in which the requirement of same zero dynamics may be enforced. The first way is to force the equivalent output to have the same zero dynamics at every set point.

Definition 1: Consider system (1). The outputs y and $\mathrm{y}^{*}$ are said to be globally dynamically equivalent if $\mathrm{h}(\mathrm{x})$ and $\mathrm{h}^{*}(\mathrm{x})$ have the same level sets, i.e.

$$
\forall y_{0} \exists y_{0}^{*} \text { such that } l_{y_{0}}=l_{y_{0}^{*}}
$$

and

$$
\forall y_{0}^{*} \exists y_{0} \text { such that } l_{y_{v}}=l_{y_{0}^{*}} \text {. }
$$

An immediate consequence of the definition is that there is a real function of a real variable $\varphi$ such that

$$
h(x)=\varphi\left[h^{*}(x)\right] .
$$

Indeed, the above definition of dynamic equivalence establishes the existence of a bijection $\varphi$ from $h^{*}\left(\mathbf{R}^{\prime \prime}\right)$ onto $h\left(\mathbf{R}^{n}\right)$ such that

$$
l_{\varphi\left(y_{0}^{*}\right)}=l_{y_{0}^{*}} \quad \forall y_{0}^{*}
$$

and

$$
l_{y_{0}}=l_{q^{-t}\left(y_{0}\right)} \quad \forall y_{0}
$$

hence eq. (4).

Another immediate consequence of this definition is the following. Consider the augmented system given in eq. (1) with $y$ and $y^{*}$ globally dynamically equivalent, and let $\mathscr{E}$ be the equilibrium set

$$
\mathscr{E}=\left\{x \in \mathbf{R}^{n} \mid \exists \lambda \in \mathbf{R}: f(x)+\lambda g(x)=0\right\} .
$$

Furthermore, take $y_{0}$ such that

$$
I=l_{\nu_{0}} \cap \mathscr{E} \neq \emptyset \text {. }
$$

By definition, the (unforced) zero dynamics is the dynamics of the system when the output is constrained to a constant value (Byrnes and Isidori, 1985). When $y=y_{0}$ or $y^{*}=y_{0}^{*}=\varphi^{-1}\left(y_{0}\right)$, the states of the system will evolve on the same manifold. Since the choice of output does not affect the state equations, the $u-y$ and $u-y^{*}$ systems clearly have the same (unforced) zero dynamies for every $y_{0}$, locally around every element of $I$.

An example illustrating the use of a globally dynamically equivalent output is the measurement of flow through an orifice plate. The flowrate, $F$, is proportional to the square root of the pressure drop across the plate, $\Delta P$, which is the measured output

$$
F \propto(\Delta P)^{1 / 2} \text {. }
$$

A globally dynamically equivalent output for this system is the square root of the measurement, i.e.

$$
y^{*}=(\Delta P)^{1 / 2}
$$

This example can be found in Shinskey's book on process control (1988). Another example is pH pro- 
cesses when the modeling is done in terms of titration curves, as developed in Wright and Kravaris (1991) and Wright et al. (1991).

The other method by which a dynamically equivalent output may be defined is to require the equivalent output to have the same (unforced) zero dynamics only at the set point value of the process output. Thus, for each value of the set point, it is possible to have a different equivalent output defined.

Definition 2: Consider system (1). Given $\mathrm{y}_{0}$ and $\mathrm{y}_{0}^{*}$, the outputs $\mathrm{y}$ and $\mathrm{y}^{*}$ are said to be pointwise dynamically equivalent at $\mathrm{y}_{0}$ and $\mathrm{y}_{0}^{*}$, respectively, if the corresponding level sets are equal:

$$
l_{y_{\mathrm{o}}}=l_{y_{\bar{q}}^{*}}
$$

An immediate consequence of this definition is that the (unforced) zero dynamics of the $u-y$ and $u-y^{*}$ systems are guaranteed to be the same at the particular points $y_{0}$ and $y_{0}^{*}$, locally around every element of I.

A more intuitive interpretation of the notion of dynamic equivalence arises from the observation that the set point value of the output forces the states to have a certain relationship with respect to each other, as defined by the output map. A dynamically equivalent output for the system at that set point is one that forces the states to have the same relationship with respect to each other.

Remark 1: Note that for linear output functions, the notions of global and pointwise dynamic equivalence clearly coincide.

\section{DYNAMIC EQUIVALENCE TO A LINEAR OUTPUT}

Dynamically equivalent outputs are useful in the case of a linear or nearly linear system with a nonlincar state/output map. For such systems, if a dynamically equivalent output can be defined which is linear with respect to the state variables, a linear controller may be used. Consider the system

$$
\begin{aligned}
& \dot{x}=A x+b u \\
& y=h(x) .
\end{aligned}
$$

Regulating the output, $y$, to a given set point value, $y_{0}$, will enforce a certain relationship between the states, $x$, given by $h(x)=y_{0}$. The question is whether that relationship is linear. From Definition 1, global dynamic equivalence to a linear output simply means that $h$ is a function of a linear combination of the states

$$
h\left(x_{1}, \ldots, x_{n}\right)=\varphi\left(c_{1} x_{1}+\cdots+c_{n} x_{n}\right)
$$

where $\varphi$ is a real function of a real variable and $c_{1}, \ldots, c_{n}$ are real numbers. The following theorem gives necessary and sufficient conditions that must be met for a globally dynamically equivalent output to exist and means for calculating the coefficients.
Theorem 1: Let $X$ be an open, connected set in $\mathbf{R}^{n}$ representing the operating region and $\mathrm{h}(\mathrm{x})$ be $a$ welldefined and smooth scalar function on $\mathrm{X}$. Assume that

$$
\frac{\partial h}{\partial x}(x) \neq 0 \quad \text { for every } x \in X
$$

Then $\mathrm{h}(\mathrm{x})$ is globally dynamically equivalent to $\mathrm{y}^{*}=\mathrm{c}_{1} \mathrm{x}_{1}+\cdots+\mathrm{c}_{n} \mathrm{x}_{n}$ if and only if

$$
\frac{\frac{\partial h}{\partial x_{j}}(x)}{\frac{\partial h}{\partial x_{n}}(x)}=\text { constant }=\frac{c_{l}}{c_{n}} \quad \forall j=1,2, \ldots, n-1
$$

where the index $\mathrm{n}$ is such that

$$
\frac{\partial h}{\partial x_{n}}(x) \neq 0
$$

Proof: Assume that $h\left(x_{1}, \ldots, x_{n}\right)=\varphi\left(c_{1} x_{1}+\cdots\right.$ $\left.+c_{n} x_{n}\right)$. The partial of $h$ with respect to $x_{i}$ is given by

$$
\frac{\partial h}{\partial x_{j}}=\varphi^{\prime}\left(c_{1} x_{1}+\cdots+c_{n} x_{n}\right) c_{j} \quad \forall j=1,2, \ldots, n
$$

Then

$$
\frac{\frac{\partial h}{\partial x_{j}}}{\frac{\partial h}{\partial x_{n}}}=\frac{c_{j}}{c_{n}} \quad \forall j=1,2, \ldots, n-1
$$

Assuming that the partial differential equations given in eq. (11) hold, a particular solution is

$$
h(x)=c_{1} x_{1}+\cdots+c_{n} x_{n}
$$

The general solution is therefore given by

$$
h\left(x_{1}, \ldots, x_{n}\right)=\varphi\left(c_{1} x_{1}+\cdots+c_{n} x_{n}\right)
$$

and this shows that $h(x)$ is globally dynamically equivalent to

$$
y^{*}=c_{1} x_{1}+\cdots+c_{n} x_{n}
$$

The case of pointwise dynamic equivalence to a linear system is also interesting and useful in applications. The following proposition gives necessary and sufficient local conditions which must be met in order for a linear pointwise dynamically equivalent output to exist for every set point value of $y_{0}$.

Theorem 2: Let $\mathrm{X}$ be an open, connected set in $\mathbf{R}^{n}$ representing the operating region and $\mathrm{h}(\mathrm{x})$ be $a$ welldefined and smooth scalar function on $\mathrm{X}$. Assume that

$$
\frac{\partial h}{\partial x}(x) \neq 0 \text { for every } x \in X \text {. }
$$


Then the level sets of $\mathrm{h}$ will be locally planar around every point of $\mathrm{X}$ if and only if

Rank $\left[\begin{array}{ccccc}0 & \frac{\partial h}{\partial x_{1}}(x) & \frac{\partial h}{\partial x_{2}}(x) & \cdots & \frac{\partial h}{\partial x_{n}}(x) \\ \frac{\partial h}{\partial x_{1}}(x) & \frac{\partial^{2} h}{\partial x_{1}^{2}}(x) & \frac{\partial^{2} h}{\partial x_{1} \partial x_{2}}(x) & \cdots & \frac{\partial^{2} h}{\partial x_{1} \partial x_{n}}(x) \\ \frac{\partial h}{\partial x_{2}}(x) & \frac{\partial^{2} h}{\partial x_{2} \partial x_{1}}(x) & \frac{\partial^{2} h}{\partial x_{2}^{2}}(x) & \cdots & \frac{\partial^{2} h}{\partial x_{2} \partial x_{n}}(x) \\ \vdots & \vdots & \vdots & \\ \frac{\partial h}{\partial x_{n}}(x) & \frac{\partial^{2} h}{\partial x_{n} \partial x_{1}}(x) & \frac{\partial^{2} h}{\partial x_{n} \partial x_{2}}(x) & \cdots & \frac{\partial^{2} h}{\partial x_{n}^{2}}(x)\end{array}\right]=2$

for every $\mathrm{x} \in \mathrm{X}$.

Proof: It must be shown that for a given arbitrary $y_{0} \in h(X)$ and $x^{\circ} \in l_{y_{0}}, l_{y_{0}} \cap \mathbf{N}\left(x^{0}\right)$ is planar, where $\mathbf{N}\left(x^{0}\right)$ denotes a neighborhood of $x^{0}$, if and only if (12) is satisfied for every $x \in l_{y_{0}} \cap \mathbf{N}\left(x^{0}\right)$. By appropriate permutation of indices, set $n$ to be an index for which

$$
\frac{\partial h}{\partial x_{n}}\left(x^{0}\right) \neq 0 \text {. }
$$

Then $h\left(x_{1}, x_{2}, \ldots, x_{n}\right)=y_{0}$ is uniquely solvable for $x_{n}$ in a neighborhood of $x^{0}$. This defines a smooth implicit function

$$
x_{n}=\Gamma\left(x_{1}, x_{2}, \ldots, x_{n-1}\right)
$$

locally around $x^{a}$. Clearly, $h\left(x_{1}, x_{2}, \ldots, x_{n}\right)=y_{0}$ will represent a hyperplane if and only if $\Gamma\left(x_{1}, x_{2}, \ldots, x_{n-1}\right)$ is a linear function, i.e.

$$
\frac{\partial^{2} \Gamma}{\partial x_{i} \partial x_{j}}\left(x_{1}, x_{2}, \ldots, x_{n-1}\right)=0
$$

for all $i=1,2, \ldots, n-1$ and $j=1,2, \ldots, n-1$.

Applying the implicit function theorem to eq. (13) yields

$$
\begin{aligned}
\frac{\partial \Gamma}{\partial x_{i}}\left(x_{1}, \ldots, x_{n-1}\right)= \\
-\frac{\partial h}{\partial x_{i}}\left[x_{1}, \ldots, x_{n-1}, \Gamma\left(x_{1}, \ldots, x_{n-1}\right)\right] \\
\frac{\partial h}{\partial x_{n}}\left[x_{1}, \ldots, x_{n-1}, \Gamma\left(x_{1}, \ldots, x_{n-1}\right)\right]
\end{aligned}
$$

Differentiating eq. (15) with respect to $x_{j}$ and using eq. (15) results in

$$
\frac{\partial^{2} \Gamma}{\partial x_{i} x_{j}}=\frac{\frac{\partial h}{\partial x_{i}} \frac{\partial h}{\partial x_{n}} \frac{\partial^{2} h}{\partial x_{j} x_{n}}+\frac{\partial h}{\partial x_{j}} \frac{\partial h}{\partial x_{n}} \frac{\partial^{2} h}{\partial x_{i} x_{n}}-\left(\frac{\partial h}{\partial x_{n}}\right)^{2} \frac{\partial^{2} h}{\partial x_{i} x_{j}}-\frac{\partial h}{\partial x_{i}} \frac{\partial h}{\partial x_{j}} \frac{\partial^{2} h}{\partial x_{n}^{2}}}{\left(\frac{\partial h}{\partial x_{n}}\right)^{3}}
$$


Equivalently, for every fixed $j$ between 1 and $n-1$, are linearly dependent for every $j$. Equivalently, the the matrix matrix

$$
\left[\begin{array}{ccc}
0 & \frac{\partial h}{\partial x_{j}} & \frac{\partial h}{\partial x_{n}} \\
\frac{\partial h}{\partial x_{1}} & \frac{\partial^{2} h}{\partial x_{1} \partial x_{j}} & \frac{\partial^{2} h}{\partial x_{1} \partial x_{n}} \\
\frac{\partial h}{\partial x_{2}} & \frac{\partial^{2} h}{\partial x_{2} \partial x_{j}} & \frac{\partial^{2} h}{\partial x_{2} \partial x_{n}} \\
\vdots & \vdots & \vdots \\
\frac{\partial h}{\partial x_{i}} & \frac{\partial^{2} h}{\partial x_{i} \partial x_{j}} & \frac{\partial^{2} h}{\partial x_{i} \partial x_{n}} \\
\vdots & \vdots & \vdots \\
\frac{\partial h}{\partial x_{n}} & \frac{\partial^{2} h}{\partial x_{n} \partial x_{j}} & \frac{\partial^{2} h}{\partial x_{n}^{2}}
\end{array}\right]
$$

does not have full rank, i.e. the column vectors $\left[\begin{array}{ccccccc}0 & \frac{\partial h}{\partial x_{1}} & \frac{\partial h}{\partial x_{2}} & \cdots & \frac{\partial h}{\partial x_{j}} & \cdots & \frac{\partial h}{\partial x_{n}} \\ \frac{\partial h}{\partial x_{1}} & \frac{\partial^{2} h}{\partial x_{1}^{2}} & \frac{\partial^{2} h}{\partial x_{1} \partial x_{2}} & \cdots & \frac{\partial^{2} h}{\partial x_{1} \partial x_{j}} & \cdots & \frac{\partial^{2} h}{\partial x_{1} \partial x_{n}} \\ \frac{\partial h}{\partial x_{2}} & \frac{\partial^{2} h}{\partial x_{2} \partial x_{1}} & \frac{\partial^{2} h}{\partial x_{2}^{2}} & \cdots & \frac{\partial^{2} h}{\partial x_{2} \partial x_{j}} & \cdots & \frac{\partial^{2} h}{\partial x_{2} \partial x_{n}} \\ \vdots & \vdots & \vdots & & \vdots & & \vdots \\ \frac{\partial h}{\partial x_{n}} & \frac{\partial^{2} h}{\partial x_{n} \partial x_{1}} & \frac{\partial^{2} h}{\partial x_{n} \partial x_{2}} & \cdots & \frac{\partial x^{2} h}{\partial x_{n} \partial x_{j}} & \cdots & \frac{\partial^{2} h}{\partial x_{n}^{2}}\end{array}\right]$

has at most two linearly independent columns. Finally, it is easy to see that the above matrix cannot have Rank $=1$ or be identically zero since it would correspond to a constant $h(x)$ (zero gradient for all $x$ ). Hence the result.

Remark 2: When the condition (12) is satisfied for each different $y_{0}$, a different linear output, in general, is obtained.

Remark 3: This theorem implies that for every $y_{0}, l_{y_{0}} \cap X$ are planes. In some situations, the condition (12) may be satisfied only at a particular $l_{y_{0}}$. A pointwise dynamically equivalent output then may be defined only for that particular point $y_{0}$.

Remark 4: From the point of view of checking the conditions in particular applications, it is more convenient to use conditions (17) where the $n$th coordinate is such that

$$
\frac{\partial h}{\partial x_{n}} \neq 0 .
$$

A simple code in a symbolic mathematics package can be used to check these conditions for a particular $h(x)$.

Remark 5: It is clear from eq. (17) that by interchanging $i$ and $j$ the same condition is obtained. For this reason the number of independent conditions is $n(n-1) / 2$.

Remark 6: A globally dynamically equivalent output satisfying theorem 1 will also satisfy theorem 2 . However, a pointwise dynamically equivalent output satisfying theorem 2 will not in general satisfy theorem 1 .

Remark 7: In general, the coefficients $c_{i}$ for a pointwise dynamically equivalent linear output must be calculated numerically. If $\boldsymbol{h}$ satisfies theorem 2 , then $\Gamma$ is linear. Given the setpoint, $y_{0}$, it is straightforward from eq. (13) to calculate points on the $(n-1)$-dimensional plane and the coefficients that describe it. 
Corollary: Let $\mathrm{X}$ be an open, connected set in $\mathbf{R}^{n}$ and $\mathbf{Y}$ an open, connected set in $\mathbf{R}$. Suppose the output is an implicit function of the states, i.e.

$$
H(x, y)=0
$$

where $\mathbf{H}$ is a well-defined smooth function which is uniquely solvable in $\mathrm{y}$ for every $\mathrm{x} \in \mathbf{X}$. Assume that

$$
\frac{\partial H}{\partial x}(x, y) \neq 0 \quad \forall(x, y) \in X \times Y .
$$

Then the level sets of $\mathbf{H}$ will be locally planar around every point in $\mathrm{X} \times \mathrm{Y}$ if and only if
Consider the following output:

$$
y=\left[\frac{\alpha x_{1}+\beta x_{2}}{\gamma x_{1}+\delta x_{2}}\right]^{k} .
$$

It is straightforward to verify that this output map satisfies the condition (12). Given an arbitrary positive set point value, $y=y_{0}$, a pointwise dynamically equivalent output for the system is

$$
y^{*}-\left[\alpha-\gamma\left(y_{0}\right)^{1 / k}\right] x_{1}+\left[\beta-\delta\left(y_{0}\right)^{1 / k}\right] x_{2}
$$

for every $(x, y) \in X \times Y$.

Proof: The proof of the corollary goes along the same lines as the proof of theorem 2. Selecting $n$ to be an index for which

$$
\frac{\partial H}{\partial x_{n}}(x, y) \neq 0
$$

an implicit function is defined

$$
x_{n}=\Gamma\left(x_{1}, x_{2}, \ldots, x_{n-1} ; y_{0}\right)
$$

as the solution of

$$
H\left(x_{1}, x_{2}, \ldots, x_{n} ; y_{0}\right)=0 .
$$

The level sets of $H$ will be planar if and only if, for every $y_{0}$,

$$
\frac{\partial^{2} \Gamma}{\partial x_{i} \partial x_{j}}\left(x_{1}, x_{2}, \ldots, x_{n-1}\right)=0
$$

for all $i=1,2, \ldots, n-1$ and $j=1,2, \ldots, n-1$.

But

$\frac{\partial^{2} \Gamma}{\partial x_{i} x_{j}}=\frac{\frac{\partial H}{\partial x_{i}} \frac{\partial H}{\partial x_{n}} \frac{\partial^{2} H}{\partial x_{j} x_{n}}+\frac{\partial H}{\partial x_{j}} \frac{\partial H}{\partial x_{n}} \frac{\partial^{2} H}{\partial x_{i} x_{n}}-\left(\frac{\partial H}{\partial x_{n}}\right)^{2} \frac{\partial^{2} H}{\partial x_{i} \partial x_{j}}-\frac{\partial H}{\partial x_{i}} \frac{\partial H}{\partial x_{j}} \frac{\partial^{2} H}{\partial x_{n}^{2}}}{\left(\frac{\partial H}{\partial x_{n}}\right)^{3}}$ and this leads to eq. (21) following the same argument as in the proof of theorem 2.

The use of pointwise dynamically equivalent outputs is best illustrated through two simple examples. with the set point value

$$
y_{0}^{*}=\mathbf{0} .
$$

Finally, as is shown in detail in Wright and Kravaris (1991), all $\mathrm{pH}$ processes modeled in terms of concentrations where the chemical equilibria are fast can be described by

$$
\begin{gathered}
\dot{x}=A x+b u \\
10^{-y}-10^{y-14}+\sum_{i=1}^{n} a_{i}(y) x_{i}=0
\end{gathered}
$$

where the output map is implicitly defined by eq. (30). The conditions of the corollary are satisfied as the second partials of the outpul map are all zero. It is easy to see that a pointwise dynamically equivalent output exists and is given by

$$
y^{*}=-\sum_{i=1}^{n} a_{i}\left(y_{0}\right) x_{i}
$$

with set point value

$$
\left(y_{0}^{*}\right)_{\mathrm{sp}}=10^{-y_{\mathrm{v}}}-10^{\left(y_{\mathrm{o}}-14\right)}
$$

for every set point value $y_{0}$ of $y$. Thus the dynamically equivalent output makes it possible to design a linear 
controller based on the linear $u-y^{*}$ system without the nonlinear static gain of the original process. For these systems, the equivalent output has the added physical interpretation of being the strong acid equivalent of the system.

\section{USING DYNAMICALLY EQUIVALENT OUTPUTS IN SHM LINEARIZABLE SYSTEMS}

The use of SHM state feedback to stabilize a nonlinear CSTR has been demonstrated in Hoo and Kantor $(1985,1986)$. In this section, conditions are given for the existence of a dynamically equivalent output for a SHM linearized system. Consider a nonlinear SISO system of the form

$$
\begin{aligned}
& \dot{x}=f(x)+g(x) u \\
& y=h(x)
\end{aligned}
$$

that satisfies the following conditions:

$\left(^{*}\right)$ the vector fields $g(x), \operatorname{ad}_{f}^{1} g(x), \ldots, \operatorname{ad}_{f}^{n-1} g(x)$ are linearly independent

$\left.{ }^{* *}\right)$ the set of vector fields $\left\{g(x), \operatorname{ad}^{1} g(x), \ldots\right.$, $\left.\operatorname{ad}_{f}^{n-2} g(x)\right\}$ is involutive

Then the system of $n-1$ first-order partial differential equations

$$
\begin{gathered}
\sum_{i=1}^{n} g_{i}(x) \frac{\partial w}{\partial x_{i}}=0 \\
\sum_{i=1}^{n}\left(\operatorname{ad}_{f}^{1} g\right)_{i}(x) \frac{\partial w}{\partial x_{i}}=0 \\
\vdots \\
\sum_{i=1}^{n}\left(\operatorname{ad}_{f}^{n-2} g\right)_{i}(x) \frac{\partial w}{\partial x_{i}}=0
\end{gathered}
$$

admits a nontrivial solution, $w=q(x)$, and the transformation (Hunt et al., 1983)

$$
\xi=T(x)=\left[\begin{array}{c}
q(x) \\
L_{f} q(x) \\
\vdots \\
L_{f}^{n-1} q(x)
\end{array}\right]
$$

is invertible and transforms the dynamic system of eq. (33) into the natural coordinate form

$$
\begin{aligned}
\dot{\xi}_{1}= & \xi_{2} \\
\dot{\xi}_{2}= & \xi_{3} \\
& \vdots \\
\dot{\xi}_{n-1}= & \xi_{n} \\
\dot{\xi}_{n}= & {\left[L_{f}^{n} q(x)\right]_{x=T^{-1}(\xi)}+\left[L_{g} L_{f}^{n-1} q(x)\right]_{x=T^{-1}(\xi)} u } \\
y= & {[h(x)]_{x=T^{-1}(\xi) .} }
\end{aligned}
$$

When this system is subject to the state feedback

$u=\frac{v-L_{f}^{n} q(x)-\alpha_{1} L_{f}^{n-1} q(x)-\cdots-\alpha_{n-1} L_{f} q(x)-\alpha_{n} q(x)}{L_{g} L_{f}^{n-1} q(x)}$ where $\alpha_{1}, \ldots, \alpha_{n-1}, \alpha_{n}$ are scalar adjustable parameters, then in closed loop

$$
\begin{aligned}
\dot{\xi}_{1}= & \xi_{2} \\
\dot{\xi}_{2}= & \xi_{3} \\
& \vdots \\
\dot{\xi}_{n-1}= & \xi_{n} \\
\dot{\xi}_{n}= & -\alpha_{n} \xi_{1}-\alpha_{n-1} \xi_{2}-\cdots-\alpha_{1} \xi_{n}+v \\
y= & h\left(T^{-1}(\xi)\right)
\end{aligned}
$$

which is a linear controllable system with characteristic polynomial

$$
s^{n}+\alpha_{1} s^{n-1}+\cdots+\alpha_{n-1} s+\alpha_{n}
$$

but a nonlinear state/output map. Note that eq. (38) is of the form eq. (9) discussed in the previous section.

The output of the system (38) will be linear in $\xi$-coordinates if (Tarn et al., 1987)

$$
\mathrm{d} h(x) \in \mathbf{R}-\operatorname{span}\left\{\mathrm{d} q(x), \mathrm{d} L_{f} q(x), \ldots, \mathrm{d} L_{f}^{n-1} q(x)\right\}
$$

i.e.

$$
\mathrm{d} h(x)\left[\begin{array}{c}
\mathrm{d} q(x) \\
\mathrm{d} L_{f} q(x) \\
\vdots \\
\mathrm{d} L_{f}^{n-1} q(x)
\end{array}\right]^{-1}=\text { constant. }
$$

Note that the above condition is extremely restrictive; it can only be satisfied when $r=n$ or the forced zero dynamics of (38) are linear. As a means around this restriction, the notion of distinguished outputs was proposed by Kantor (1987). A distinguished output is defined as any output that corresponds to $r=n$. Such an output clearly meets the conditions given above for total linearization [the choice $q(x)=h(x)$ satisfies (34) and (40)]. However, distinguished outputs will, in general, not be equivalent to the given original output of the system and, because they induce maximal relative order, will ensure the most indirect effect of the manipulated input on the output.

Using the notion of dynamically equivalent ouputs, it is still possible to achieve the purpose of total linearization for the original system output. If $h \circ T^{-1}$ is nonlinear but dynamically equivalent to a linear output, that dynamically equivalent linear output can be used in the control structure. The conditions under which this can happen will now be derived.

Remark 8: Glohal dynamic equivalence in the transformed coordinates implies

$$
h\left(T^{-1}(\xi)\right)=\varphi\left(c_{1} \xi_{1}+c_{2} \xi_{2}+\cdots+c_{n} \xi_{n}\right) .
$$

Thus, a globally dynamically equivalent output exists 
for a system of the form of eq. (38) if and only if $h(x)=\varphi\left(c_{1} q(x)+c_{2} L_{f} q(x)+\cdots+c_{n} L_{f}^{n-1} q(x)\right)$
Proof: Since it is desired to have pointwise dynamic equivalence in terms of the transformed coordinates, let $\bar{h}=h\left(T^{-1}(\xi)\right)$, then from theorem 1 of the previous section

$$
\operatorname{Rank}\left[\begin{array}{ccccc}
0 & \frac{\partial \bar{h}}{\partial \xi_{1}}(\xi) & \frac{\partial \bar{h}_{2}}{\partial \xi_{2}}(\xi) & \cdots & \frac{\partial \bar{h}}{\partial \xi_{n}}(\xi) \\
\frac{\partial \bar{h}}{\partial \xi_{1}}(\xi) & \frac{\partial^{2} \bar{h}}{\partial \xi_{1}^{2}}(\xi) & \frac{\partial^{2} \bar{h}}{\partial \xi_{1} \partial \xi_{2}}(\xi) & \cdots & \frac{\partial^{2} \bar{h}}{\partial \xi_{1} \partial \xi_{n}}(\xi) \\
\frac{\partial \bar{h}}{\partial \xi_{2}}(\xi) & \frac{\partial^{2} \bar{h}}{\partial \xi_{2} \partial \xi_{1}}(\xi) & \frac{\partial^{2} \bar{h}}{\partial \xi_{2}^{2}}(\xi) & \cdots & \frac{\partial^{2} \bar{h}}{\partial \xi_{2} \partial \xi_{n}}(\xi) \\
\vdots & \vdots & \vdots & & \vdots \\
\frac{\partial \bar{h}}{\partial \xi_{n}}(\xi) & \frac{\partial^{2} \bar{h}}{\partial \xi_{n} \partial \xi_{1}}(\xi) & \frac{\partial^{2} \bar{h}}{\partial \xi_{n} \partial \xi_{2}}(\xi) & \cdots & \frac{\partial^{2} \bar{h}}{\partial \xi_{n}^{2}}(\xi)
\end{array}\right]=2
$$

where $\varphi$ is an arbitrary function. Necessary and sufficient conditions for the existence of a globally dynamically equivalent output and a means of calculating the coefficients are straightforward from theorem 1.

The following proposition gives necessary and sufficient local conditions that must be met in order for a linear pointwise dynamically equivalent output to exist for a system of the form eq. (38).

Theorem 3: Let $\mathrm{X}$ be an open, connected set and $\mathrm{h}(\mathrm{x})$ be a well-defined and smooth scalar function on X. Assume. that

$$
\frac{\partial h}{\partial x}(x) \neq 0
$$

for every $\mathrm{x} \in \mathrm{X}$, and that the conditions (*) and (*) are satisfied for every $\mathrm{x} \in \mathrm{X}$. Denote by $\mathrm{q}(\mathrm{x})$ a nontrivial solution of the set of PDEs (34), and by $\mathrm{T}(\mathrm{x})$ the vector function defined by eq. (35). Then the level sets of $\mathrm{h} \circ \mathrm{T}^{-1}$ will be locally planar around every point of $\mathrm{T}^{-1}(\mathrm{X})$ if and only if

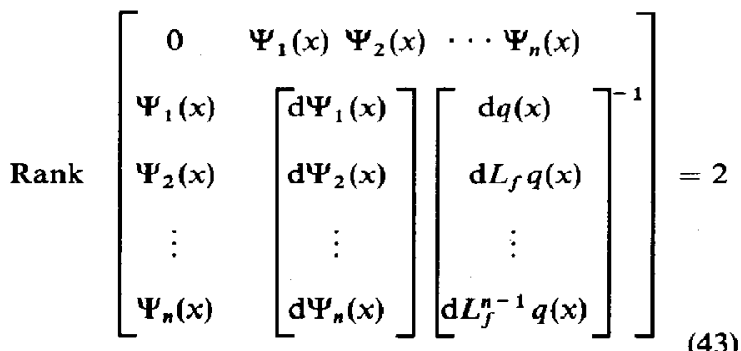

for every $x$ in $X$, where

$\left[\begin{array}{ll}\Psi_{1}(x) & \Psi_{2}(x) \cdots \Psi_{n}(x)\end{array}\right]=\mathrm{d} h(x)\left[\begin{array}{c}\mathrm{d} q(x) \\ \mathrm{d} L_{f} q(x) \\ \vdots \\ \mathrm{d} L_{f}^{n-1} q(x)\end{array}\right]^{-1}$
The first row and first column of this matrix contain 0 and the gradient of $\bar{h}(\xi)$. The remaining matrix is the Hessian of $\bar{h}(\xi)$. The gradient of $\bar{h}(\xi)$ is given by

$$
\mathrm{d} \bar{h}(\xi)=\left[\mathrm{d} h(x)\left(\frac{\partial T}{\partial x}\right)^{-1}\right]
$$

or

$$
\mathrm{d} \bar{h}(\xi)=\left[\mathrm{d} h(x)\left[\begin{array}{c}
\mathrm{d} q(x) \\
\mathrm{d} L_{f} q(x) \\
\vdots \\
\mathrm{d} L_{f}^{m-1} q(x)
\end{array}\right]^{-1}\right]_{x=T^{-1}(\xi)}
$$

Set

$$
\mathrm{d} h(x)\left[\begin{array}{c}
\mathrm{d} q(x) \\
\mathrm{d} L_{f} q(x) \\
\vdots \\
\mathrm{d} L_{f}^{n-1} q(x)
\end{array}\right]=\left[\begin{array}{l}
\Psi_{1}(x) \\
\Psi_{2}(x) \cdots \Psi_{n}(x)
\end{array}\right]
$$

The Hessian of $\bar{h}(\xi)$ is then given by

$$
\begin{aligned}
& \operatorname{Hessian}[\bar{h}(\xi)]=\left[\begin{array}{c}
\mathrm{d}\left[\frac{\partial \bar{h}}{\partial \xi_{1}}\right](\xi) \\
\mathrm{d}\left[\frac{\partial \bar{h}}{\partial \xi_{2}}\right](\xi) \\
\vdots \\
\mathrm{d}\left[\frac{\partial \bar{h}}{\partial \xi_{n}}\right](\xi)
\end{array}\right] \\
& =\left[\begin{array}{c}
\mathrm{d}\left[\Psi_{1} \circ T^{-1}\right](\xi) \\
\mathrm{d}\left[\Psi_{2} \circ T^{-1}\right](\xi) \\
\vdots \\
\mathrm{d}\left[\Psi_{0} \circ T^{-1}\right](\xi)
\end{array}\right]
\end{aligned}
$$




$$
\begin{aligned}
& =\left[\left[\begin{array}{c}
\mathrm{d} \Psi_{1}(x) \\
\mathrm{d} \Psi_{2}(x) \\
\vdots \\
\mathrm{d} \Psi_{n}(x)
\end{array}\right]\left(\frac{\partial T}{\partial x}(x)\right)^{-1}\right. \\
& =\left[\left[\begin{array}{c}
\mathrm{d} \Psi_{1}(x) \\
\mathrm{d} \Psi_{2}(x) \\
\vdots \\
\mathrm{d} \Psi_{n}(x)
\end{array}\right]\left[\begin{array}{c}
\mathrm{d} q(x) \\
\mathrm{d} L_{f} q(x) \\
\vdots \\
\mathrm{d} L_{f}^{n-1} q(x)
\end{array}\right]\right.
\end{aligned}
$$

Combining the gradient and the Hessian leads directly to the result.

Remark 9: The Tarn et al: (1987) condition

$$
\mathrm{d} h(x)\left[\begin{array}{c}
\mathrm{d} q(x) \\
\mathrm{d} L_{f} q(x) \\
\vdots \\
\mathrm{d} L_{f}^{n-1} q(x)
\end{array}\right]^{-1}=\text { constant }
$$

or

$$
\left[\Psi_{1}(x) \quad \Psi_{2}(x) \cdots \Psi_{n}(x)\right]=\text { constant }
$$

is clearly a special case of the proposition.

The controller structures used for combining the SHM linearizing transformation with a dynamically equivalent output are shown in Figs 2 4. If state feedback is possible, the structure used with either a globally dynamically equivalent output or a pointwise dynamically equivalent output is as shown in Fig. 2. If state measurements are not available and output feedback is used, the resulting structure differs depending on whether the equivalent output is globally or pointwise dynamically equivalent. From eq. (41) it is clear that a globally dynamically equivalent output is of the form

$$
y=\varphi\left(y^{*}\right) .
$$

Thus, a state estimator is not required in the calculation of the equivalent output and the output feedback structure shown in Fig. 3 may be used. For pointwise dynamically equivalent outputs, the structure shown in Fig. 4 would be used.

\section{Example}

The control method is illustrated through the following example. Consider a CSTR with the irreversible, first-order reaction

$$
\boldsymbol{A} \rightarrow \boldsymbol{B}
$$

The system is governed by the equations

$$
\begin{aligned}
\frac{\mathrm{d} C_{A}}{\mathrm{~d} t} & =\frac{F}{V}\left(C_{A \mathrm{in}}-C_{A}\right)-k(T) C_{A} \\
\frac{\mathrm{d} T}{\mathrm{~d} t} & =\frac{F}{V}\left(T_{\mathrm{in}}-T\right)+k(T) C_{A} \frac{(-\Delta H)}{\rho c_{p}}+\frac{Q}{\rho c_{p} V}
\end{aligned}
$$

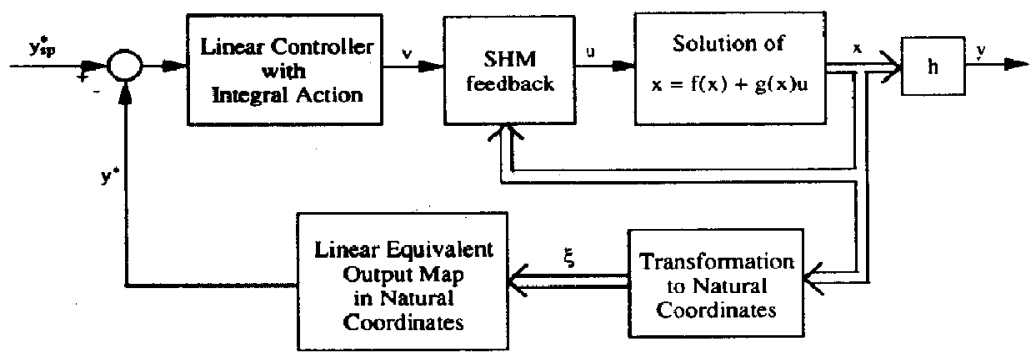

Fig. 2. State feedback controller structure for use with SHM feedback and dynamically equivalent outputs.

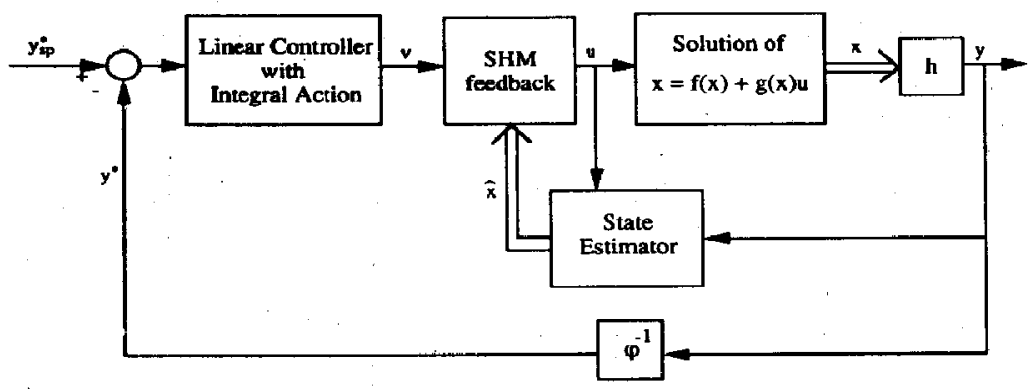

Fig. 3. Output feedback controller structure for use with SHM feedback and globally dynamically equivalent outputs. 


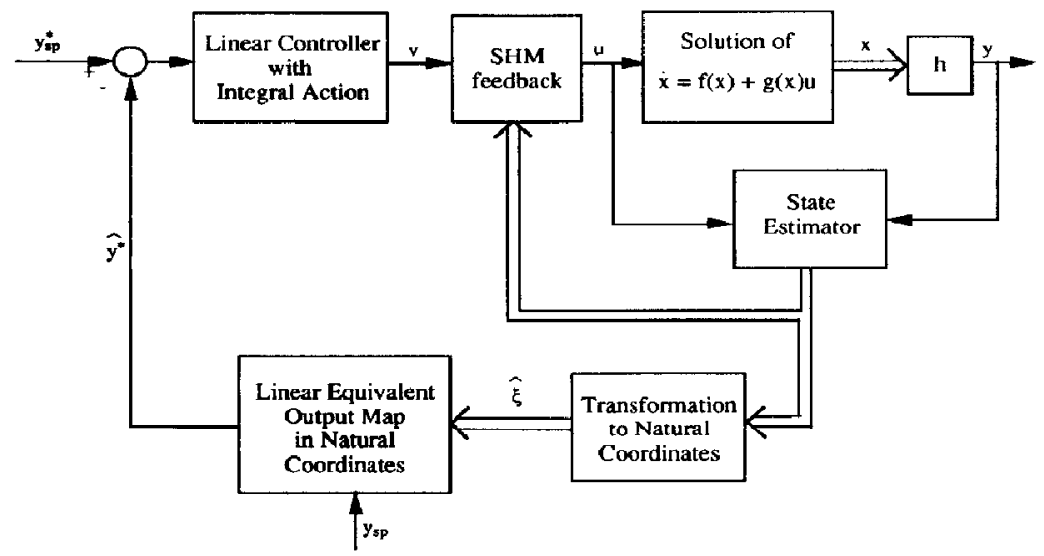

Fig. 4. Output feedback controller structure for use with SHM feedback and pointwise dynamically equivalent outputs.

where

$$
k(T)=A_{0} \mathrm{e}^{-E / R T}
$$

the manipulated input is $u=Q$

the controlled output is $y=T$.

A transformation to natural coordinates is obtained by setting the first coordinate equal to any function of $C_{A}$. The simplest transformation is given by

$$
\begin{aligned}
& \xi_{1}=C_{A} \\
& \xi_{2}=\frac{F}{V}\left(C_{A_{\text {in }}}-C_{A}\right)-k(T) C_{A} .
\end{aligned}
$$

The SHM state feedback is then given by

$$
u=\frac{v-L_{f}^{2} q\left(\xi_{1}, \xi_{2}\right)-\alpha_{1} \xi_{2}-\alpha_{2} \xi_{1}}{L_{g} L_{f} q\left(\xi_{1}, \xi_{2}\right)}
$$

where

$$
\begin{aligned}
L_{f}^{2} q\left(\xi_{1}, \xi_{2}\right)= & -\xi_{2}\left(\frac{F}{V}+Z\right) \\
& +\frac{A E \xi_{1}}{R} Z^{2}\left[\frac{F}{V}\left(T_{\mathrm{in}}-k^{-1}(Z)\right)\right. \\
& \left.+\frac{(-\Delta H) \xi_{1} Z}{\rho C_{p}}\right] \\
L_{g} L_{f} q\left(\xi_{1}, \xi_{2}\right)= & \frac{A E}{\rho C_{p} V R} Z^{2} \\
Z= & \frac{\frac{F}{V}\left(C_{A_{\mathrm{in}}}-\xi_{1}\right)-\xi_{2}}{\xi_{1}}
\end{aligned}
$$

and the model in natural coordinates is then

$$
\begin{aligned}
\dot{\xi}_{1} & =\xi_{2} \\
\xi_{2} & =L_{f}^{2} q\left(\xi_{1}, \xi_{2}\right)+L_{\theta} L_{f} q\left(\xi_{1}, \xi_{2}\right) u \\
y & =k^{-1}\left(\frac{\frac{F}{V}\left(C_{A_{\text {in }}}-\xi_{1}\right)-\xi_{2}}{\xi_{1}}\right)
\end{aligned}
$$

Thus, the state equations have been made linear by the feedback, but the output is nonlincar. This is true regardless of the transformation chosen in eq. (52). Notice that eq. (54) is of the same type as the first example in the previuus section [eq. (26)]. It is straightforward to verify that the rank condition (12) holds for the output map in eq. (54). A linear pointwise dynamically equivalent output for the process in natural coordinates is

$$
y^{*}=k\left(T_{\mathrm{sp}}\right) \xi_{1}-\frac{F}{V}\left(C_{A_{\text {in }}}-\xi_{1}\right)+\xi_{2} .
$$

In the original coordinates (55) becomes

$$
y^{*}=\left[k\left(T_{\mathrm{sp}}\right)-k(T)\right] C_{A} .
$$

Physically, controlling the equivalent output to $y_{\mathrm{sp}}^{*}=0$ has the obvious interpretation of controlling the reaction rate.

Discrete-time simulations were performed using the parameter values given in Table 1 . At the given conditions, the reactor exhibits three steady states. The desired steady state for operation is the middle, or open-loop unstable one. For the simulations that follow, the SHM state feedback is tuned so that the resulting system follows first-order (linear) dynamics in $y^{*}$ with the time constant $1 / \lambda$. This corresponds to choosing

$$
\begin{aligned}
& \alpha_{1}=k\left(T_{\mathrm{sp}}\right)+\frac{F}{V}+\lambda \\
& \alpha_{2}=\left(k\left(T_{\mathrm{sp}}\right)+\frac{F}{V}\right) \lambda .
\end{aligned}
$$

The first disturbance studied is an unmeasured $5^{\circ}$ change in the inlet temperature. The open-loop responses in $T$ and $y^{*}$ are shown in Figs 5 and 6 , respectively. Notice the nonlinear behavior exhibited in these figures and that the relatively small inlet disturbance has a large effect on the final steady state. For this simulation study, only the temperature is assumed to be measured; a reduced-order state observer that simulates the first equation of eq. (51) is 
Table 1. Parameter values used in simulations

$$
\begin{aligned}
& C_{A I}=1.5 \mathrm{kmol} \mathrm{m}^{-3} \\
& A_{0}=1.5 \times 10^{6} \mathrm{~m}^{3} \mathrm{kmol}^{-1} \mathrm{~s}^{-1} \\
& E=58,000 \mathrm{~kJ} \mathrm{kmol}^{-1} \mathrm{~K}^{-1} \\
& \rho=1000 \mathrm{~kg} \mathrm{~m}^{-3} \\
& C_{p}=1 \mathrm{~kJ} \mathrm{~kg}^{-1} \mathrm{C}^{-1} \\
& F=0.1 \mathrm{~m}^{3} \mathrm{~s}^{-1}
\end{aligned}
$$

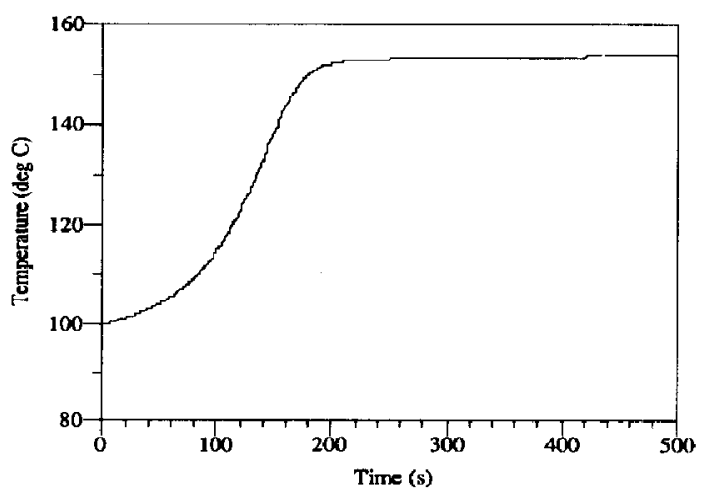

Fig. 5. Open-loop temperature response for $5^{\circ}$ change in inlet temperature.

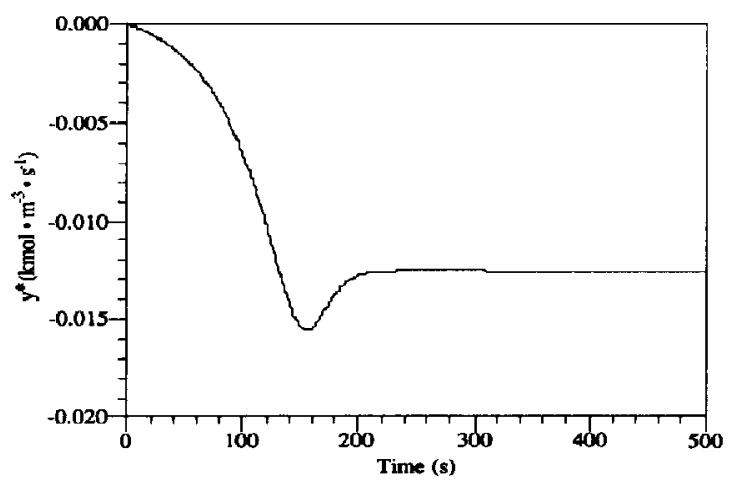

Fig. 6. Open-loop response in $y^{*}$ for $5^{\circ}$ change in inlet temperature.

used for concentration. The control structure employed is that of Fig. 4 with the external controller being a PI in terms of $y^{*}$. The closed-loop simulation results for $T, y^{*}$, and $Q$, using $K_{c}=0.02$ and $\lambda=1 / \tau_{1}=0.03$ are shown in Figs $7-9$, respectively. These results show a fast response in $y^{*}$ and a return, without offset, to the set point for both $y^{*}$ and $T$.

Another set of runs was performed where the disturbance was an increase in the inlet concentration from 1.5 to $1.6 \mathrm{kmol} \mathrm{m}^{-3}$. The control simulations for this case assume that all state measurements are available. The open-loop responses for reactor temperature, outlet concentration and $y^{*}$ are shown in Figs 10-12, respectively. Ayain nolice the resulting nonlinear behavior and the large difference in final steady state.

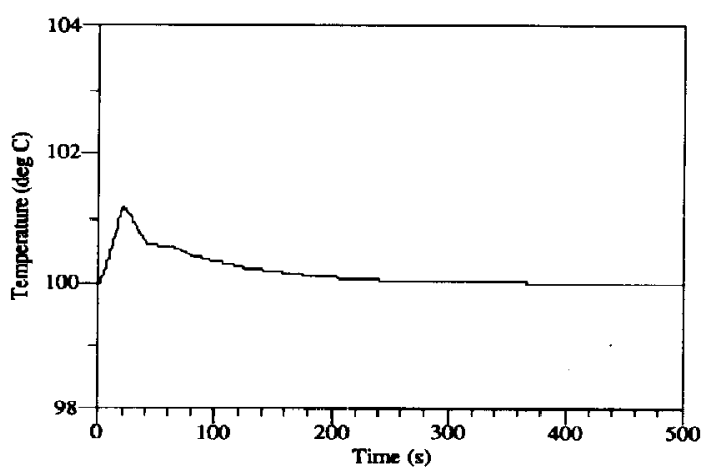

Fig. 7. Closed-loop temperature response for $5^{\circ}$ change in inlet temperature.

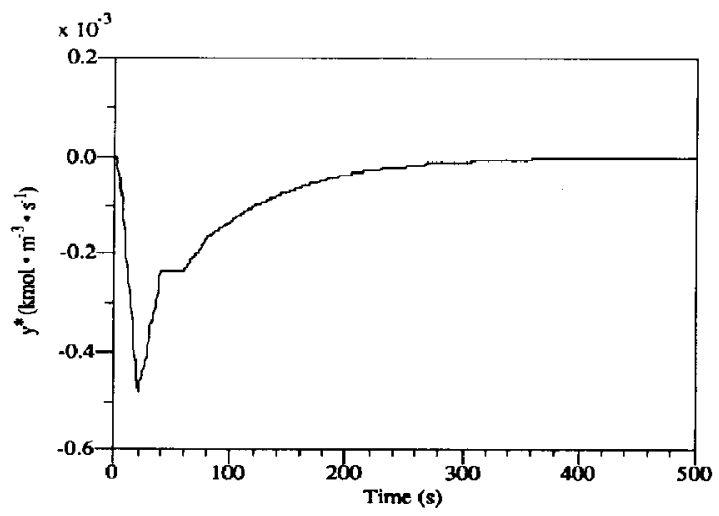

Fig. 8. Closed-loop response in $y^{*}$ for $5^{\circ}$ change in inlet temperature.

Two different external linear PI controllers were used. The first uses Kantor's distinguished output, which for this example is $C_{A}$. The second uses the pointwise dynamically equivalent output (56). The results using the distinguished output are shown in Figs 13-16. The tuning parameters for this run are $K_{c}=0.0005$ and $\lambda=1 / \tau_{1}=0.03$. Figure 13 shows the reactor temperature response. Note that there is a $2.4^{\circ}$ offset in the final value. This results from the fact that $T$ and $C_{A}$ are not dynamically equivalent. The response of $C_{A}$ is shown in Fig. 14. Notice that $C_{A}$ is controlled back to its initial point, which is its set point value. The changes in the manipulated input, $Q$, are shown in Fig. 15. The resulting trajectory of $y^{*}$ is shown in Fig. 16. Notice that a similar offset to the one noted in $T$ is exhibited in this figure. 


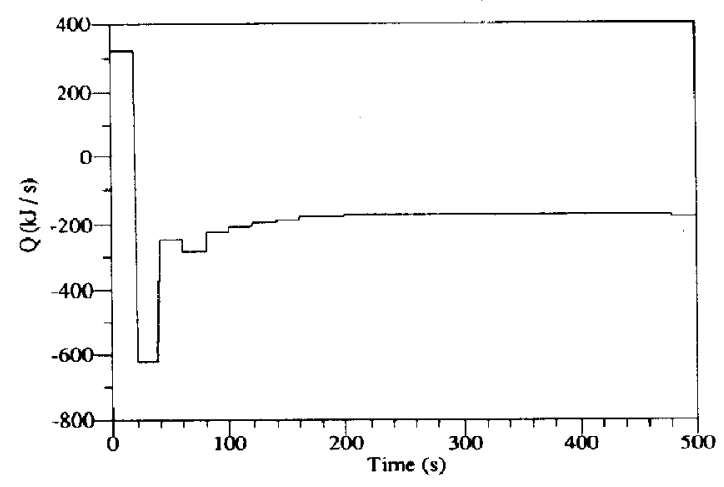

Fig. 9. Changes in $Q$ in response to a $5^{\circ}$ change in inlet temperature.

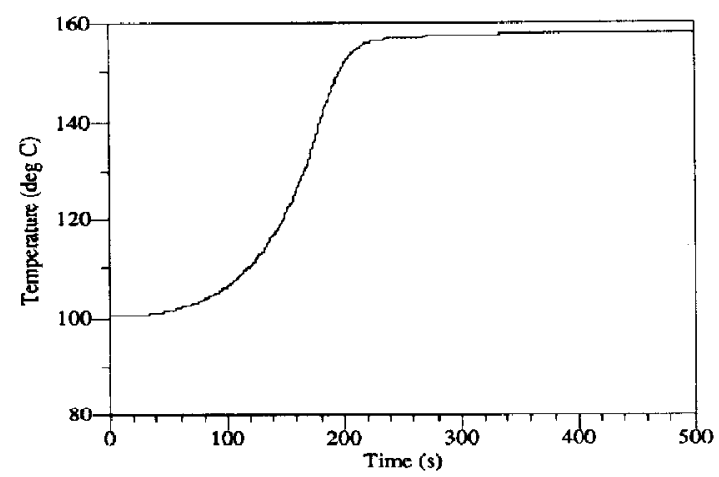

Fig. 10. Open-loop temperature response for inlet concentration disturbance.

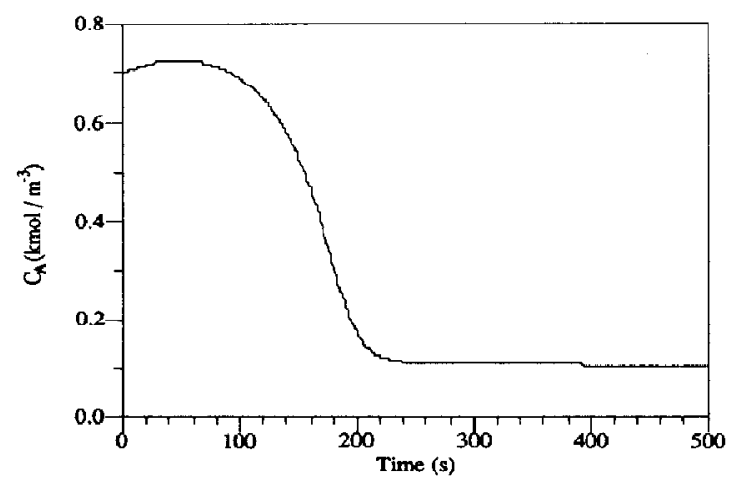

Fig. 11. Open-loop response in $C_{A}$ for inlet concentration disturbance.

Simulation results using the pointwise dynamically equivalent output in the state feedback structure of Fig. 2 are given in Figs 17-20. The external controller was again a PI. The PI and SHM tuning parameters are $K_{c}=0.02$ and $\lambda=1 / \tau_{1}=0.03$. Figure 17 shows the response in reactor temperature. The correspond-

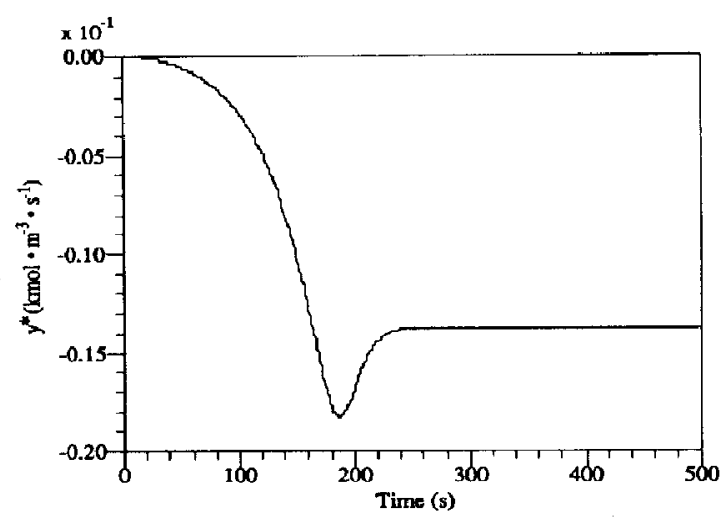

Fig. 12. Open-loop response in $y^{*}$ for inlet concentration disturbance.

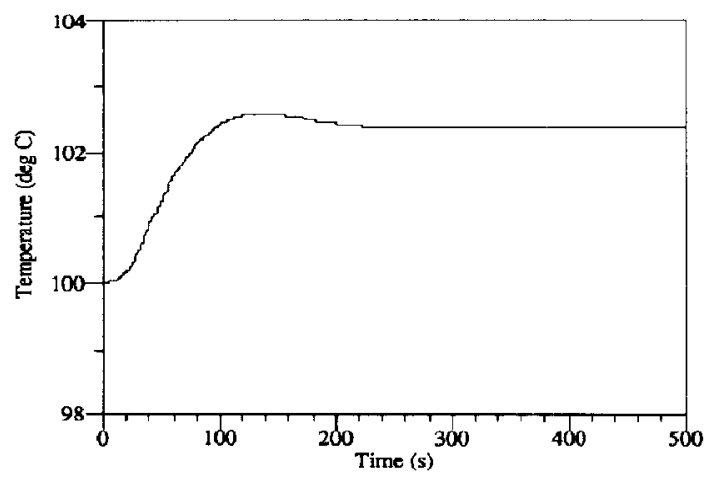

Fig. 13. Closed-loop temperature response using distinguished output.

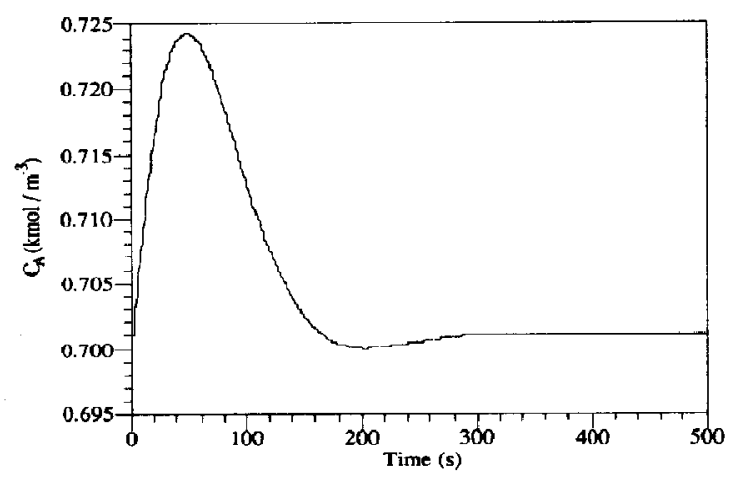

Fig. 14. Closed-loop response in concentration using distinguished output.

ing $y^{*}$ profile is shown in Fig. 18. Notice that there is no offset from set point in the final steady state in either of these values. The changes in the manipulated input, $Q$, are shown in Fig. 19. The response of $C_{A}$ is shown in Fig. 20. As is expected, when there is no final offset in $T$ and $y^{*}$, there is a difference between the 


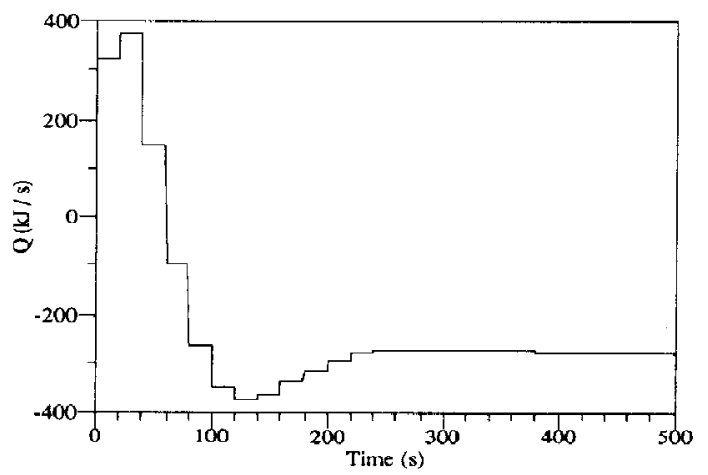

Fig. 15. Changes in $Q$ using distinguished output.

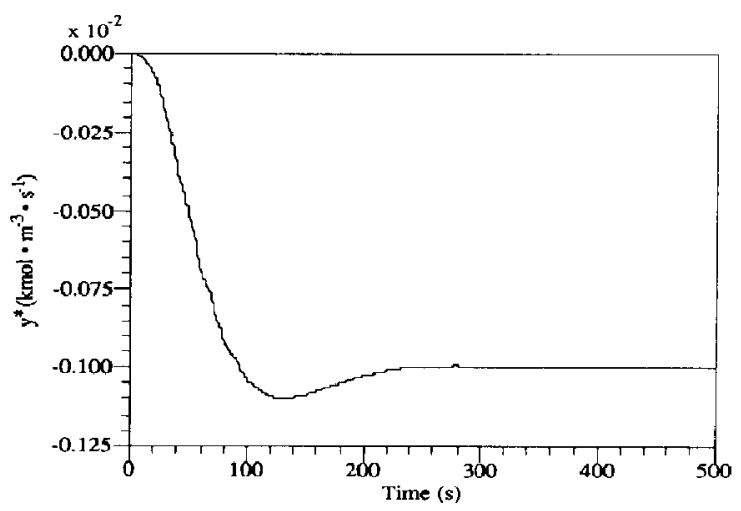

Fig. 16. Closed-loop response in $y^{*}$ using distinguished out. put.

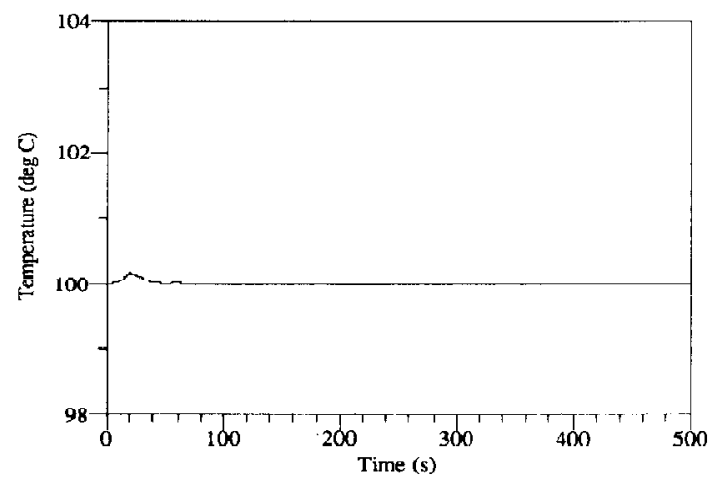

Fig. 17. Closed-loop temperature response using dynamically equivalent output.

initial and final values of $C_{A}$. This is again due to the nonequivalence of $T$ and $C_{A}$. The main conclusion to draw from these simulation runs is that, when choosing an output to simplify the controller synthesis problem, extreme care must be taken to ensure that the original control problem will also be satisfied. The concerns that must be addressed are fully answered in the notion of dynamically equivalent outputs.

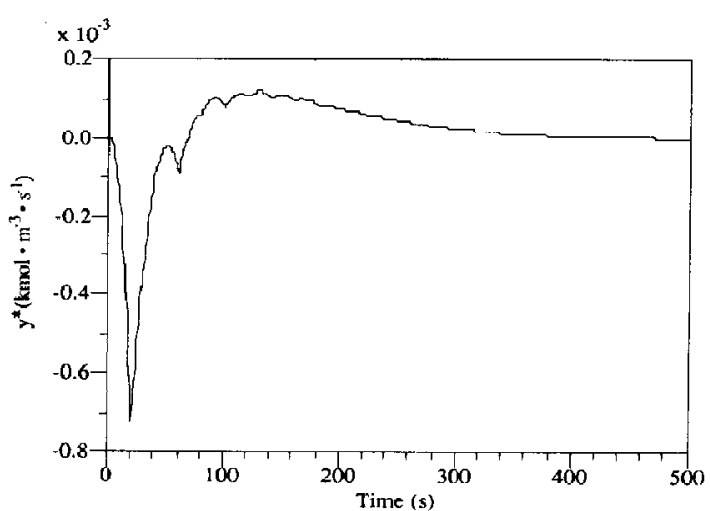

Fig. 18. Closed-loop response in $y^{*}$ using dynamically equivalent output.

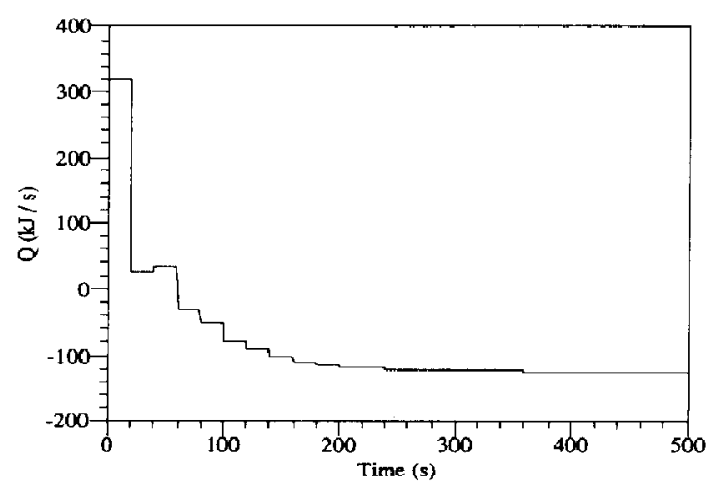

Fig. 19. Changes in $Q$ using dynamically equivalent output.



Fig. 20. Closed-loop response in $C_{A}$ using dynamically equivalent output.

\section{CONTROLLER SYNTHESIS FOR NONLINEAR CLOSED-LOOP INPUT/OUTPUT DYNAMICS}

In the previous sections, systems with nearly linear dynamics, such as $\mathrm{pH}$ processes, or SHM linearizable dynamics, such as the CSTR example, were examined. For such systems it is natural to look for a dynamically equivalent output that is linear to simplify the 


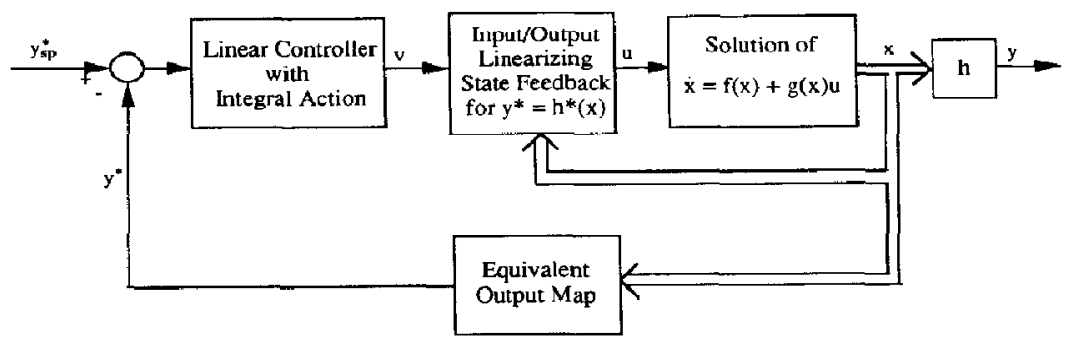

Fig. 21. State fecdback controller structure for use with GLC feedback and dynamically equivalent outputs.

controller synthesis problem. The resulting controller structure provides a response that is linear in terms of the dynamically equivalent output, but nonlinear in terms of the system's original output. Viewed from a different perspective, a dynamically equivalent output is actually a means of designing a nonlinear controller to provide a specified nonlinear closed-loop response. This idea will be explored in this section for general nonlinear systems.

Controller design methods for nonlinear processes are typically based on some notion of linearity that is enforced in closed loop (input-to-state linearity or input-to-output linearity). This is done for convenience since it is the linear dynamics that are wellunderstood and for which controller performance specifications can easily be expressed. If nonlinear dynamics are requested in closed loop, this will automatically imply that the nonlinearity of the closedloop dynamics is tunable, i.e. there will be, in essence, an infinite number of tunable parameters. If closedloop input/output dynamics which are nonlinear in the output are still sought, they must be "linear in something else" in order to have a finite number of tunable parameters. The quantity with respect to which the closed-loop dynamics is requested to be linear must be a function of the process variables, i.e. the states. It will be advantageous to synthesize a controller in terms of this quantity whenever the quantity is more important on physical grounds than the actual measured output. Consider the general system

$$
\begin{aligned}
\dot{x} & =f(x)+g(x) u \\
y & =h(x) \\
y^{*} & =h^{*}(x)
\end{aligned}
$$

where $h^{*}(x)$ is the function of the states with respect to which the dynamics must be linear. Nonlinear closedloop dynamics that are linear with respect to $h^{*}(x)$ will be of the form

$$
h^{*}(x)+\sum_{k} \gamma_{k} \frac{\mathrm{d}^{k}}{\mathrm{~d} t^{k}}\left[h^{*}(x)\right]=y_{\mathrm{sp}}^{*}
$$

where $y_{\mathrm{sp}}^{*}$ is the desired set point value of $y^{*}$ and the $\gamma_{k}$ are tunable parameters. Assuming closed-loop stability, the system will eventually reach $h^{*}\left(x_{s}\right)=y_{s p}^{*}$, where $x_{s}$ is a process steady state. If $h^{*}$ is dynamically equivalent to $h$, this automatically implies that $h\left(x_{s}\right)=y_{\text {sp }}$ even if the equilibrium line is shifted away from its nominal position (due to persistent changes in the process disturbances), and therefore the original output is still controlled to its set point value.

This suggests a method of synthesizing a controller for nonlinear processes that will have nonlinear closed-loop dynamics. A GLC controller (Kravaris and Chung, 1987) is used to regulate $y^{*}=h^{*}(x)$ to its set point. The input/output linearizing state feedback is given by

$$
u=\frac{v-h^{*}(x)-\beta_{1} L_{f} h^{*}(x)-\cdots-\beta_{r^{*}} L_{j}^{*} h^{*}(x)}{\beta_{r^{*}} L_{g} L_{f}^{r^{*}-1} h^{*}(x)}
$$

where $r^{*}$ is the relative order of the $u-y^{*}$ system and $\beta_{1}, \ldots, \beta_{r^{*}}$ are tunable scalar parameters. This will give the following closed-loop response in terms of $y^{*}$ :

$$
y^{*}+\beta_{1} \frac{\mathrm{d} y^{*}}{\mathrm{~d} t}+\cdots+\beta_{r^{*}} \frac{\mathrm{d}^{*^{*}} y^{*}}{\mathrm{~d} t^{r^{*}}}=v
$$

The closed-loop response in terms of $y$ will be nonlinear. The combination of the dynamically equivalent output and the control law represents a nonlinear controller designed to provide a nonlinear response. The dynamically equivalent output is the tool through which this can be accomplished.

The controller structures used for obtaining nonlinear closed-loop dynamics in the original system output are shown in Figs 21-23. If state feedback is possible, the structure used with either a globally dynamically equivalent output or a pointwise dynamically equivalent output is as shown in Fig. 22. If state measurements are not available and output feedback is used, the resulting structure differs depending on whether the equivalent output is globally or pointwise dynamically equivalent. As was the case when combining SHM state feedback with globally dynamically equivalent outputs, a state estimator is not required in the calculation of the equivalent output. Thus, the output feedback structure shown in Fig. 22 may be used. For pointwise dynamically equivalent outputs, a state estimator is required to compute the equiva- 


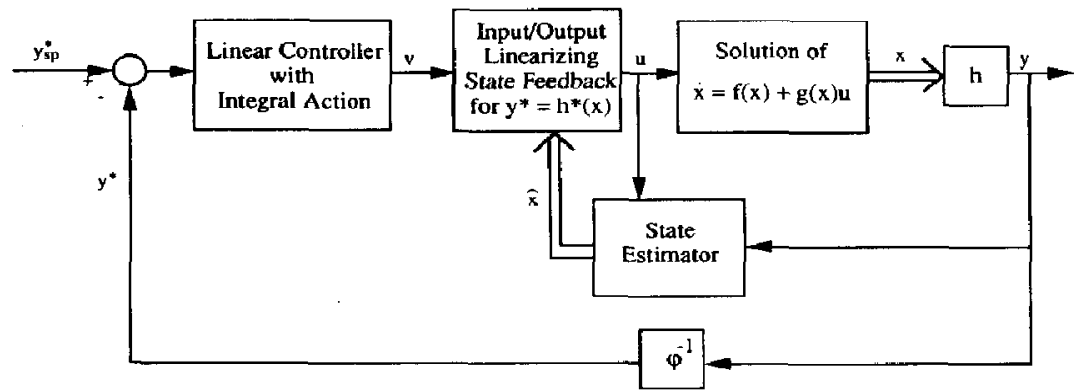

Fig. 22. Output feedback controller structure for use with GLC feedback and globally dynamically equivalent outputs.

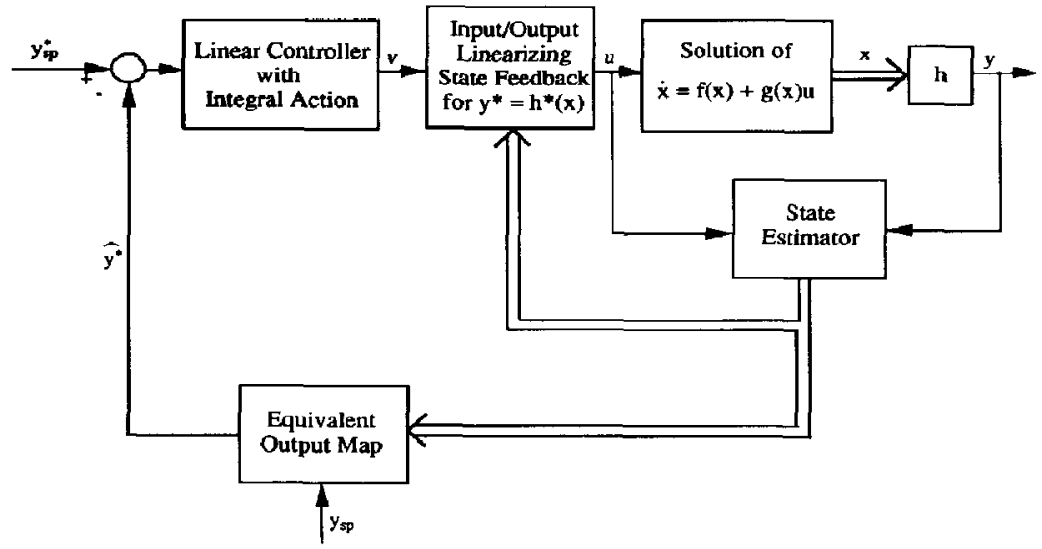

Fig. 23. Output feedback controller structure for use with GLC feedback and pointwise dynamically equivalent outputs.

lent output on-line and the structure shown in Fig. 23 would be used.

\section{Example}

Consider a system similar to that of the last section but with a second-order reaction, i.e. CSTR with the irreversible, second-order reaction

$$
\boldsymbol{A} \rightarrow \boldsymbol{B} \text {. }
$$

The system is governed by the equations

$$
\begin{aligned}
\frac{\mathrm{d} C_{A}}{\mathrm{~d} t} & =\frac{F}{V}\left(C_{A_{\mathrm{in}}}-C_{A}\right)-k(T) C_{A}^{2} \\
\frac{\mathrm{d} T}{\mathrm{~d} t} & =\frac{F}{V}\left(T_{\mathrm{in}}-T\right)+k(T) C_{A}^{2} \frac{(-\Delta H)}{\rho c_{p}}+\frac{Q}{\rho c_{P} V}
\end{aligned}
$$

where the manipulated input is $u=Q$ and the controlled output is $y=T$.

The state equations may still be linearized by a SHM state feedback, but the nonlinear output in the transformed coordinates will not be dynamically equivalent to a linear output (the rank condition of theorem 1 is not satisfied). Assume that from product quality considerations it is more important to control the rate of reaction than the measured temperature.

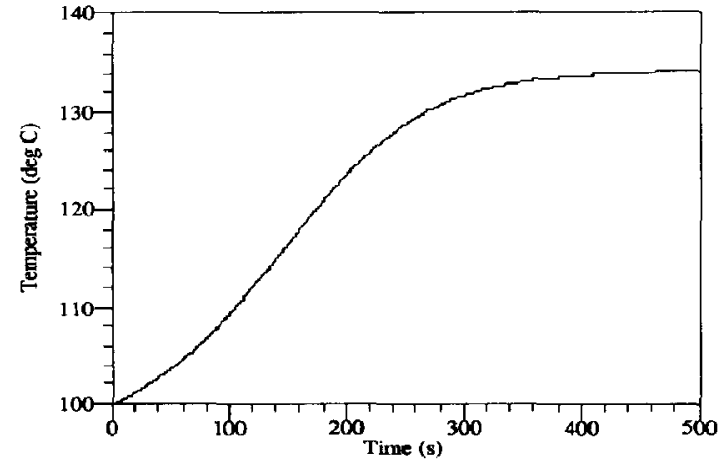

Fig. 24. Open-loop temperature response in second reactor example.

Motivated by the example of the previous section, one can define the alternate output

$$
y^{*}=\left[k\left(T_{\mathrm{sp}}\right)-k(T)\right] C_{A}^{2}
$$

which is clearly dynamically equivalent to the original output. The control methodology outlined previously 


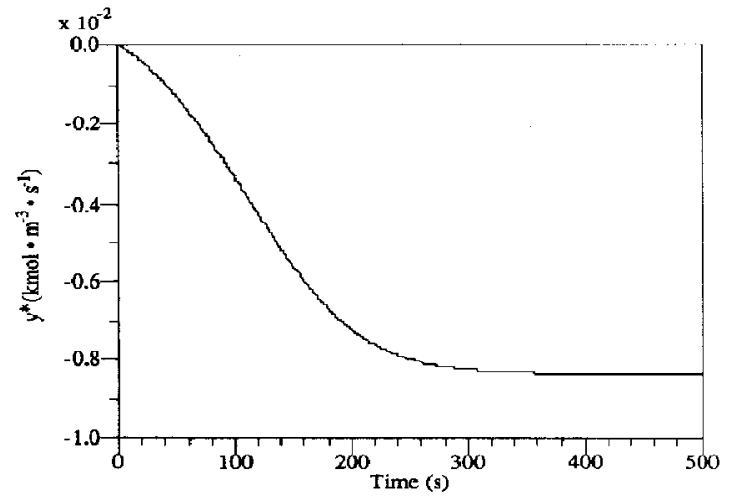

Fig. 25. Open-loop response in $y^{*}$ for second reactor example.

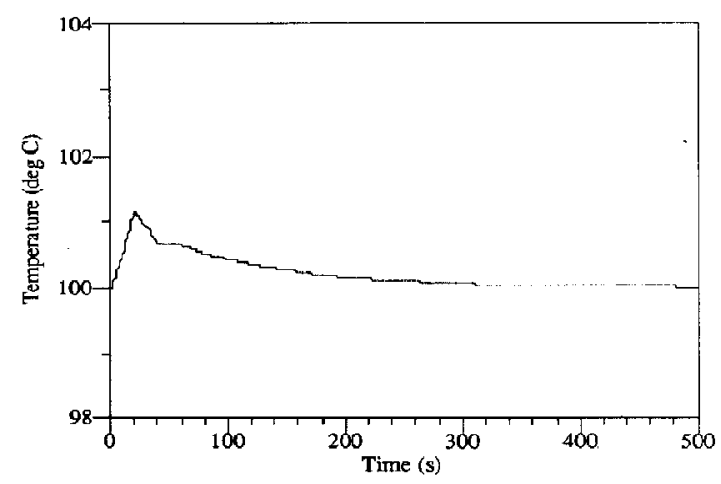

Fig. 26. Closed-loop temperature response for second reactor example.

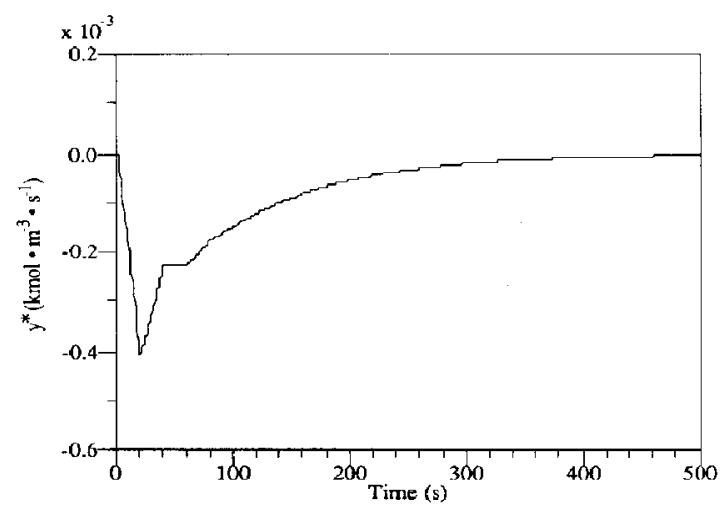

Fig. 27. Closed-loop response in $y^{*}$ for second reactor example.

with $y^{*}$ given by eq. (61) as output can then be applied. The response of $T$ will be nonlinear.

Discrete-time simulations were performed using the same conditions as given previously in Table 1 . The disturbance used is the unmeasured $5^{\circ}$ step in inlet temperature. The open-loop responses in reactor tem-

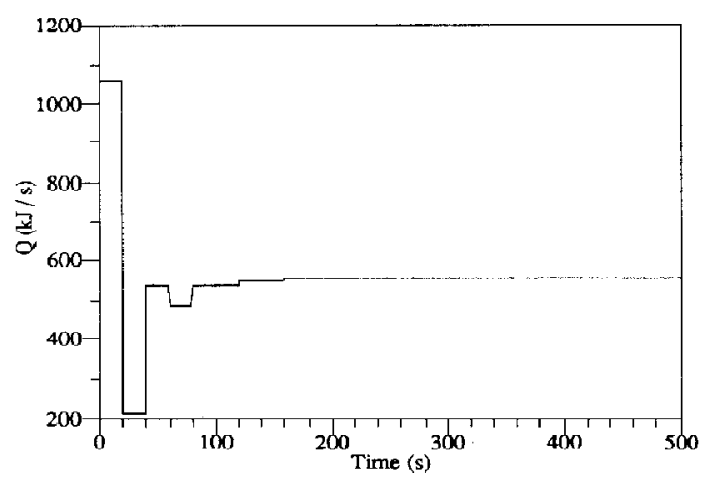

Fig, 28. Closed-loop changes in $Q$ in second reactor example.

perature and $y^{*}$ are shown in Figs 24 and 25, respectively. The closed-loop responses using the control structure of Fig. 23 are shown in Figs 26-28. The controller parameter settings for this run are $K_{c}=0.3$, $\beta_{0}=1, \beta_{1}=\tau_{1}=25$. Notice the equivalence of returning $y^{*}$ and $T$ to their respective set point values. These results demonstrate the ability to design a controller for a specified nonlinear response in the primary output through a linear controller design for the dynamically equivalent output.

\section{CONCLUSIONS}

The notion of dynamically equivalent outputs has been introduced. Two types of dynamically equivalent outputs, globally and pointwise, have been mathematically defined. Conditions for dynamic equivalence to a linear output were given. This idea was shown to be useful for systems with linear dynamics and nonlinear state/output map. Dynamic equivalence to a linear output was also combined with SHM feedback for nonlinear systems with linearizable dynamics. Controller structures illustrating this combination and a simulation example demonstrating this approach were presented. The more general notion of synthesizing nonlinear controllers to provide nonlinear closedloop dynamics was then addressed. It was shown that by combining a dynamically equivalent output and GLC feedback such controllers could be designed. Another simulation example demonstrating this idea was provided.

Acknowledgements-Financial support from the Dow Chemical Company is gratefully acknowledged. We thank Daniel Burns for his helpful suggestions.

\section{NOTATION}

$\operatorname{ad}_{f, g}^{k} \quad k$ th-order Lie bracket of $f$ and $g$ $\boldsymbol{A}_{0} \quad$ pre-exponential factor used in computer simulations 


$\begin{array}{ll}A, b & \begin{array}{l}\text { matrices in standard state space descrip- } \\ \text { tion of a linear system } \\ c_{i}\end{array} \\ c_{p} & \begin{array}{l}\text { scalar constants } \\ \text { heat capacity of inlet stream in computer } \\ \text { simulation examples } \\ \text { concentration of A in the effluent stream } \\ C_{A}\end{array} \\ C_{A_{i n}} & \begin{array}{l}\text { in simulation examples } \\ \text { inlet concentration of A in simulation } \\ \text { examples } \\ \text { activation energy used in computer simu- } \\ \text { lation examples } \\ \text { the set of state variables which, for }\end{array} \\ & \begin{array}{l}\text { a given scalar constant, constitute the } \\ \text { equilibrium set } \\ \text { flowrate through an orifice plate, also } \\ \text { flowrate into CSTR in computer simula- } \\ \text { tion examples }\end{array}\end{array}$

$f(x), g(x) \quad$ vector fields that characterize the state model of a nonlinear process

$h(x)$ scalar field that determines the state/output map

$h^{*}(x)$ scalar field that determines the state/equivalent output map

$\bar{h}(\xi) \quad$ the composition function $h\left(T^{-1}(\xi)\right)$

$H(x, y)$ implicit state/output map of a nonlinear system

$k(T)$ reaction rate constants in simulation examples

$K_{c} \quad$ external PI controller gain in simulation examples

$l \quad$ level set of the output function

$L_{f}^{k} h(x) \quad k$ th-order Lie derivative of $h$ with respect to $f$

$n \quad$ order of system

$q(x) \quad$ nontrivial solution to the set of partial differential equations used in obtaining a natural coordinate representation of a nonlinear system

$Q \quad$ heat added to the reactor

$r$ relative order

$R \quad$ ideal gas constant used in computer simulation examples

$\begin{array}{ll}t & \text { time } \\ T(x) & \text { SHM transformation }\end{array}$

$T$ temperature of the CSTR in computer simulation examples

$T_{\text {in }} \quad$ temperature of the inlet stream in computer simulation examples

$s \quad$ Laplace transform variable

$u \quad$ manipulated input

$v \quad$ GLC transformed control variable

$V \quad$ volume of CSTR in simulation example

$x \quad$ vector of state variables

$y \quad$ process output

$y^{*} \quad$ equivalent output

\section{Greek letters}

$\alpha_{i}$

$\gamma_{k}$
$\Gamma$
$\begin{aligned} & \Delta P \\ & -\Delta H\end{aligned}$
$\lambda$
$\xi$
$\rho$
$\tau_{1}$
$\varphi(x)$
$\Psi_{i}(x)$

tunable scalar parameters used for nonlinear input/output dynamics

scalar function of state variables defined when state/output map is solved for the last state variable

pressure drop across an orifice plate

heat of reaction in computer simulation examples

scalar constant

states of SHM transformed system

density of the inlet stream in computer simulation examples

external PI controller reset in simulation example

scalar field of the state variables

the $\boldsymbol{n}$ functions defined as the gradient of the composition function $h\left(T^{-1}(\xi)\right)$

Other symbols

$\begin{array}{ll}\epsilon & \text { belongs to } \\ \emptyset & \text { the empty set } \\ \mathbf{R} & \text { real line } \\ \mathbf{R}^{n} & n \text {-dimensional Euclidean space }\end{array}$

subscripts

$o$

sp

particular constant value of process output set point value

\section{REFERENCES}

Byrnes, C. I. and Isidori, A., 1985, Global feedback stabilization of nonlinear systems, in Proceedings of the 24th IEEE CDC, p. 1031. Ft. Lauderdale.

Hoo, K. A. and Kantor, J. C., 1985, An exothermic continuous stirred tank reactor is feedback equivalent to a linear system. Chem. Engng Commun. 37, 1.

Hoo, K. A. and Kantor, J. C., 1986, Linear feedback equivalence and control of an unstable biological reactor. Chem. Engng Commun. 46, 385.

Hunt, L. R., Su, R. and Meyer, G., 1983, Global transformations of nonlinear systems. IEEE Trans. autom. Control 28, 24.

Kantor, J. C., 1987, An overview of nonlinear geometric methods for process control, in Shell Process Control Workshop (Edited by D. M. Prett and M. Morari), p. 225. Butterworth, London.

Kravaris, C. and Chung, C. B., 1987, Nonlinear feedback synthesis by global input/output linearization. A.I.Ch.E.J. 33, 592.

Shinskey, F. G., 1988, Process Control Systems. McGrawHill, Maidenhead.

Tarn, T. J., Cheng, D. and Isidori, A., 1987, Pfaffian basis for affine nonlinear systems, in Proceedings of the 26th IEEE $C D C$, p. 493. Los Angeles.

Wright, R. A., 1990, Equivalent output formulations of nonlinear control problems. Ph.D. dissertation, The University of Michigan, Ann Arbor, MI.

Wright, R. A. and Kravaris, C., 1991, Nonlinear control of pH processes using the strong acid equivalent. IEC Res. 30, 1561 .

Wright, R. A., Soroush, M. and Kravaris, C., 1991, Strong acid equivalent control of pH processes: an experimental study. IEC Res. 30, 2437.

Wright, R. A. and Kravaris, C., 1992, Nonminimum phase compensation for nonlinear processes. A.I.Ch.E. J. 38, 26. 